\title{
Assigning unknown parent groups to reduce bias in genomic evaluations of final score in US Holsteins
}

\author{
S. Tsuruta, ${ }^{* 1}$ I. Misztal, ${ }^{*}$ D. A. L. Lourenco, ${ }^{*}$ and T. J. Lawlort \\ *Animal and Dairy Science Department, University of Georgia, Athens 30602 \\ †Holstein Association USA Inc., Brattleboro, VT 05301
}

\section{ABSTRACT}

Assigning unknown parent groups (UPG) in mixedmodel equations using single-step genomic BLUP was investigated to reduce bias and to increase accuracy in genomic estimated breeding values (GEBV). The original UPG were defined based on the animal's birth year and the sex of the animal's unknown parents. Combining the last 2 UPG for the animals' birth years and separating the UPG for US and non-US Holsteins were considered in the redefinition. A full data set in the 2011 national genetic evaluation of final score in US Holsteins was used to calculate estimated breeding values (EBV) for validation, and a subset of the 2011 data, which excluded phenotypes recorded after 2007, was used to calculate GEBV for all animals, including 34,500 genotyped bulls. The EBV and GEBV in 2007 were compared with EBV in the 2011 full data. The last group effects for unknown sires and dams were overestimated with the GEBV model using the reduced 2007 data. The genetic trends from EBV in 2011 and GEBV in 2007 with the original UPG in the last few years demonstrated inflation, whereas GEBV with the redefined UPG by combining the last 2 groups showed deflation. On the other hand, the redefined UPG by separating for US and non-US Holsteins reduced the bias in GEBV. Regression coefficients smaller than unity for GEBV for young genotyped bulls with no daughters in 2007 on progeny deviations in 2011 also indicated inflation. The redefining of UPG reduced bias and slightly increased accuracy in GEBV for both US and non-US genotyped bulls. Rank correlations between GEBV in 2007 and in 2011 with the redefined UPG were higher than those with no UPG and the original UPG, especially for non-US bulls. Redefining of UPG in genomic evaluation could improve reliability of GEBV and provide correct genetic trends.

Key words: unknown parent group, genomic evaluation, final score, US Holstein

Received December 11, 2013.

Accepted June 9, 2014.

${ }^{1}$ Corresponding author: shogo@uga.edu

\section{INTRODUCTION}

Unknown parent groups (UPG) can be included in mixed-model equations (MME) to account for genetic differences among defined animal groups in genetic evaluations. Ignoring genetic groups or defining poor genetic groups would introduce biased EBV. as Henderson (1974) and Kennedy (1981) noted. Westell et al. (1988) proposed rules for assigning genetic groups in animal models and proposed that a group of unknown (phantom) parents, which have no phenotypes and are selected to be parents, can be defined as a genetic group to account for genetic differences among those groups. The genetic difference could be created by a selection intensity that varies by sex, breed, selection line, trait of interest, year, region, and available amount of pedigree and genomic information. Interbull (2001) recommended using UPG according to breed, country of origin, selection path, and birth date. Currently, Holstein Association USA Inc. (Brattleboro, VT) defines UPG based on the year of birth of animals and the sex of unknown parents in the genetic evaluations for type traits in US Holsteins. Misztal et al. (2013) explained that potential bias could occur in genomic EBV (GEBV) using single-step genomic BLUP (ssGBLUP) with UPG. Tsuruta et al. (2013) reported biased UPG estimates and slow convergence in ssGBLUP for several traits in US Holsteins, Israeli Holsteins, and pigs. They redefined UPG and predicted 2 additive genetic effects from pedigree-based EBV with UPG and GEBV without UPG. The results showed that both methods reduced the bias, but that the latter required double the computer memory and triple the number of iterations to converge. When the number of phenotypes is few or zero, the poorly assigned UPG for genotyped animals may lead to a significant bias in GEBV. Biases in GEBV have been reported and discussed in several papers (Aguilar et al., 2010; Wiggans et al., 2011; Tsuruta et al., 2013) and could be due to the scale difference between pedigree-based and genomic relationships, especially for young genotyped animals. VanRaden and Wright (2013) described how preselection for genotyped animals leads to biased GEBV. Properly defined UPG 
to account for genetic differentials could reduce the bias in GEBV.

The objective of this study was to examine how to define the UPG assigned in MME to reduce bias and possibly increase accuracy in genomic evaluations for young Holstein bulls using ssGBLUP.

\section{MATERIALS AND METHODS}

A full data set from the national genetic evaluations in 2011 for final score provided by Holstein Association USA Inc. was used. The full data set consisted of 10,944,571 phenotypes for 6,586,605 cows and 9,602,031 animals in pedigree and was used for validation to predict EBV for all animals, including young bulls that had no daughters in 2007 but had daughters in 2011. A subset of the data, which excluded phenotypes of cows recorded after 2007 and consisted of 10,167,604 phenotypes and 6,012,441 cows, was used to estimate UPG effects and to predict GEBV with genotypes for 34,500 bulls, including young genotyped bulls with no daughters in 2007; that is, phenotypes and pedigrees of daughters for those young genotyped bulls used in the 2011 data were excluded to predict GEBV in 2007. The genotypes on 42,503 SNP markers were sequenced with the BovineSNP50 (50,000-SNP) BeadChip of Illumina Inc. (San Diego, CA). The animal model for national genetic evaluation for final score in US Holsteins, as described in Tsuruta et al. (2002), was applied to calculate EBV and GEBV. The BLUP90IOD program (Tsuruta et al., 2001) was used to calculate EBV and GEBV as well as UPG effects, with a convergence criterion of $10^{-14}$ as described in Tsuruta et al. (2011).

For validation of GEBV for young genotyped bulls, regression coefficients of GEBV in 2007 on progeny deviations for the 2011 data and those coefficients of determination were used as indicators of bias and realized reliability in GEBV for the 2007 data, respectively (VanRaden et al., 2009; Mäntysaari et al., 2010). Regression coefficients smaller than unity indicate that GEBV in 2007 had a larger scale than progeny deviations and that the GEBV were inflated. The regression coefficients and coefficients of determination for parent averages (PA) calculated from the 2007 data were also compared with those for GEBV.

In GEBV, the MME include

$$
\mathbf{H}^{-1}=\mathbf{A}^{-1}+\left[\begin{array}{cc}
0 & 0 \\
0 & \mathbf{G}^{-1}-\mathbf{A}_{22}^{-1}
\end{array}\right],
$$

which combines the inverse of the numerator relationship matrix (A) and the inverse of the genomic relationship matrix $(\mathbf{G})$, where $\mathbf{G}$ and $\mathbf{A}_{22}$ contain only genotyped animals and $\mathbf{H}$ is a combined matrix of $\mathbf{A}$ and G (Aguilar et al., 2010). After the QP transformation (Quaas and Pollak, 1981), the MME can be expressed as follows, ignoring other fixed effects and assuming that the additive genetic variance is equal to the residual variance, for simplicity:

$$
\left[\begin{array}{cc}
\mathbf{Z}^{\prime} \mathbf{Z}+\mathbf{H}^{-1} & -\mathbf{H}^{-1} \mathbf{Q} \\
-\mathbf{Q}^{\prime} \mathbf{H}^{-1} & \mathbf{Q}^{\prime} \mathbf{H}^{-1} \mathbf{Q}
\end{array}\right]\left[\begin{array}{c}
\mathbf{u} \\
\mathbf{g}
\end{array}\right]=\left[\begin{array}{c}
\mathbf{Z}^{\prime} \mathbf{y} \\
0
\end{array}\right]
$$

where $\mathbf{Z}$ is a design matrix that assigns phenotypes for animals; $\mathbf{Q}$ is a design matrix that assigns relationships between animals and unknown parents to UPG; $\mathbf{u}$ is a vector of genetic evaluations for animals, including UPG effects (g) and additive genetic effects (a): $\mathbf{u}=$ $\mathbf{Q g}+\mathbf{a}$; and $\mathbf{y}$ is a vector of observations (phenotypes). The design matrix $\mathbf{Q}$ can be split to 2 partitions: $\mathbf{Q}_{1}$ and $\mathbf{Q}_{2}$. We define $\mathbf{Q}_{1}$ as a matrix assigning relationships between nongenotyped animals and unknown parents to UPG, on the basis of $\mathbf{A}$. As described by Misztal et al. (2013), we define $\mathbf{Q}_{2}$ as a matrix assigning relationships for only genotyped animals to UPG, on the basis of $\mathbf{G}$, but these UPG for genotyped animals are ignored in the current ssGBLUP. Therefore, if genotyped animals have no phenotypes $\left(\mathbf{Z}^{\prime} \mathbf{Z}\right)$, the UPG for those genotyped animals are not taken into account.

The numbers of US and non-US genotyped bulls are presented in Table 1, including 26,177 US genotyped bulls and 8323 non-US genotyped bulls. The US bulls were determined by their registration numbers including characters "USA" or " 840 " for young bulls. The original UPG in the national genetic evaluation were created based on year of birth of the animal and sex of the unknown parent, combining every $4 \mathrm{yr}$ for unknown sires and every 2 yr for unknown dams (Table 2). Table 2 shows that each UPG contained 44,000 and 78,000 animals, on average, ranging from 20,000 to 52,000 sires and from 10,000 to 149,000 dams, respectively. The first redefining of UPG involved combining the last 2 UPG for unknown sires to benefit from phenotypes of other animals, even though the number of unknown sires in the last UPG was greater than the minimum size of 10 to 20 animals recommended by Interbull (2001).

All genotyped bulls had no missing parents. Table 3 shows that the number of missing parents in the pedigree file for US and non-US bulls that had daughters in 2007. Less than $1 \%$ of parents and grandparents were missing for US bulls, whereas 14\% (sires) and 19\% (dams) of parents and 16\% (paternal grandparents) and $45 \%$ (maternal grandparents) were missing for non-US bulls. Assuming that a genetic difference exists between US and non-US bulls, the second redefining of UPG involved separating US and non-US animals on the basis 
Table 1. Number of US and non-US genotyped bulls

\begin{tabular}{|c|c|c|c|}
\hline \multirow[b]{2}{*}{ Item } & \multicolumn{2}{|c|}{ Bulls } & \multirow[b]{2}{*}{ Total } \\
\hline & US & Non-US (\%) & \\
\hline \multicolumn{4}{|c|}{ Year of birth } \\
\hline$\leq 1985$ & 414 & $38(8.4)$ & 452 \\
\hline$\overline{1986}$ & 116 & $15(11.5)$ & 131 \\
\hline 1987 & 115 & $13(10.2)$ & 128 \\
\hline 1988 & 126 & $4(3.1)$ & 130 \\
\hline 1989 & 132 & $10(7.0)$ & 142 \\
\hline 1990 & 73 & $25(25.5)$ & 98 \\
\hline 1991 & 75 & $66(46.8)$ & 141 \\
\hline 1992 & 60 & $11(15.5)$ & 71 \\
\hline 1993 & 61 & $18(22.8)$ & 79 \\
\hline 1994 & 106 & $73(40.8)$ & 179 \\
\hline 1995 & 536 & $161(23.1)$ & 697 \\
\hline 1996 & 529 & $178(25.2)$ & 707 \\
\hline 1997 & 668 & $227(25.4)$ & 895 \\
\hline 1998 & 323 & $149(31.6)$ & 472 \\
\hline 1999 & 386 & $163(29.7)$ & 549 \\
\hline 2000 & 324 & $180(35.7)$ & 504 \\
\hline 2001 & 453 & $242(34.8)$ & 695 \\
\hline 2002 & 484 & $270(35.8)$ & 754 \\
\hline 2003 & 487 & $266(35.3)$ & 753 \\
\hline 2004 & 1,129 & $342(23.2)$ & 1,471 \\
\hline 2005 & 1,306 & $398(23.4)$ & 1,704 \\
\hline 2006 & 1,408 & $552(28.2)$ & 1,960 \\
\hline 2007 & 2,066 & $588(22.2)$ & 2,654 \\
\hline 2008 & 3,600 & $963(21.1)$ & 4,563 \\
\hline 2009 & 5,251 & $1,540(22.7)$ & 6,791 \\
\hline 2010 & 5,637 & $1,788(24.1)$ & 7,425 \\
\hline 2011 & 312 & $43(12.1)$ & 355 \\
\hline Total & 26,177 & $8,323(24.1)$ & 34,500 \\
\hline
\end{tabular}

of nationality in the registration number of the bulls as well as combining the last 2 groups. Table 2 also shows the number of unknown sires and dams in the redefined UPG with US and non-US parents and in the original UPG. The redefined UPG contained 37,000 sires and 8,000 dams, on average, ranging from 16,000 to 46,000 US sires and from 3,000 to 7,000 non-US sires, and from 8,000 to 148,000 US dams and from 600 to 4,000 non-US dams, respectively. Out of 34,500 genotyped bulls, 28,225 bulls had no daughters in 2007 and 3,468 bulls had daughters with phenotypes in 2011. The last UPG for 5,488 sires included many of young genotyped bulls with no daughters.

\section{RESULTS AND DISCUSSION}

Although the ssGBLUP ignores $\mathbf{Q}_{2}$ multiplied by $\mathbf{G}^{-1}-\mathbf{A}_{22}^{-1}$ for genotyped animals with no phenotypes, when $\mathbf{Q}_{2}$ or $\mathbf{G}^{-1}-\mathbf{A}_{22}^{-1}$, or both, are small, the bias of GEBV coming from the UPG will be small. Usually, $\mathbf{G}^{-1}$ is larger than $\mathbf{A}_{22}^{-1}$ when a sufficient number of animals are genotyped, with minimum errors for generations because genotypes are more informative than pedigrees, introducing more precise Mendelian sam- pling. Although all genotyped animals had known parents in this study (i.e., no UPG for genotyped animals), their parent(s) or ancestors had missing pedigrees. The majority of dams of non-US genotyped bulls had missing pedigrees, whereas US genotyped bulls could be traced back several generations. When $\mathbf{Z}^{\prime} \mathbf{Z}$ for genotyped animals is small or zero, contributions in UPG for genotyped animals from nongenotyped animals may not be negligible. If UPG effects are biased, genomic evaluations for genotyped animals could be biased, especially when nongenotyped and genotyped animals are strongly related and those genotyped animals have no phenotypes, regardless of whether their parents are known or not. In the redefinition of UPG, the last group in the original UPG formed by unknown parents for parents of young genotyped bulls was combined with the previous group to share the contributions from phenotypes (i.e., $\mathbf{Z}^{\prime} \mathbf{Z}$ ).

Figure 1 shows original UPG effects for sires estimated with the EBV model using reduced data in 2007 (EBV07) and using full data in 2011 (EBV11) with no genotypes, original UPG effects with the GEBV model using the 2007 data including all genotyped bulls (GEBV07) and including only genotyped bulls with daughters in pedigrees (GEBV07dau), and redefined UPG effects for US and non-US bulls with GEBV models using the 2007 data including all genotyped bulls (GEBV07US and GEBV07nonUS, respectively). Because all lines were identical before 1986, the figure only shows that last 5 groups between 1986 and 2002 to magnify the differences. The redefined UPG effects for GEBV07 are not shown in Figure 1 because the estimates were fully overlapped with those for original UPG effects from 1986 to 1998. The UPG effects for EBV11 and GEBV07dau models were fully overlapped, suggesting no bias in both UPG effects when all genotyped bulls had daughters with phenotypic records. The UPG estimates from the EBV11 model calculated with no young bulls that had no daughters should be unbiased. According to the agreement of these UPG estimates and assuming that they were unbiased, the last original UPG effect for the EBV07 model was more likely underestimated. Because the EBV07 model used the same pedigrees as the EBV11 model except for cows with no phenotypes in 2007, the UPG for some of the young animals had small contributions from phenotypes in 2007. As a result, the UPG declined to a similar level as 1 generation before. On the other hand, the last original UPG effect in the GEBV07 model was overestimated by 1.73 and similar to the genetic standard deviation for a final score of 1.79. Genotyped young bulls with no daughters could have affected this bias in UPG because of small contributions from progenies with phenotypes 
Table 2. Number of unknown sires and dams in redefined and original unknown parent groups

\begin{tabular}{|c|c|c|c|c|c|c|}
\hline \multirow[b]{3}{*}{ Item } & \multicolumn{3}{|c|}{ Sires } & \multicolumn{3}{|c|}{ Dams } \\
\hline & \multicolumn{2}{|c|}{ Redefined } & \multirow{2}{*}{$\begin{array}{l}\text { Original } \\
\text { (all) }\end{array}$} & \multicolumn{2}{|c|}{ Redefined } & \multirow{2}{*}{$\begin{array}{l}\text { Original } \\
\quad \text { (all) }\end{array}$} \\
\hline & US & Non-US & & US & Non-US & \\
\hline \multicolumn{7}{|l|}{ Year of birth } \\
\hline$\leq 1957$ & & & & 148,891 & 27,599 & 176,490 \\
\hline $1958-1959$ & 152,799 & 31,164 & 183,963 & 11,428 & 3,462 & 14,890 \\
\hline 1960-1961 & & & & 10,127 & 3,535 & 13,662 \\
\hline $1962-1963$ & 18,657 & 7,059 & 25,716 & 9,275 & 3,767 & 13,042 \\
\hline $1964-1965$ & & & & 8,692 & 4,285 & 12,977 \\
\hline 1966-1967 & 16,243 & 6,647 & 22,890 & 7,974 & 2,491 & 10,465 \\
\hline $1968-1969$ & & & & 8,421 & 1,880 & 10,301 \\
\hline 1970-1971 & 16,247 & 3,756 & 20,003 & 9,177 & 1,913 & 11,090 \\
\hline $1972-1973$ & & & & 10,880 & 2,130 & 13,010 \\
\hline $1974-1975$ & 17,785 & 4,897 & 22,682 & 26,783 & 2,855 & 29,638 \\
\hline $1976-1977$ & & & & 40,972 & 2,977 & 43,949 \\
\hline 1978-1979 & 22,941 & 5,445 & 28,386 & 47,158 & 2,539 & 49,697 \\
\hline 1980-1981 & & & & 54,789 & 2,043 & 56,832 \\
\hline $1982-1983$ & 27,365 & 4,238 & 31,603 & 78,814 & 2,283 & 81,097 \\
\hline $1984-1985$ & & & & 98,379 & 2,846 & 101,225 \\
\hline $1986-1987$ & 32,554 & 6,474 & 39,028 & 126,812 & 3.723 & 130.535 \\
\hline 1988-1989 & & & & 135,828 & 4,222 & 140,050 \\
\hline 1990-1991 & 23,848 & 7,367 & 31,215 & 133,419 & 3,244 & 136,663 \\
\hline $1992-1993$ & & & & 137,580 & 1,667 & 139,247 \\
\hline $1994-1995$ & 27,079 & 2,704 & 29,783 & 135,322 & 1,118 & 136,440 \\
\hline $1996-1997$ & & & & 147,593 & 1,157 & 148,750 \\
\hline 1998-1999 & 46,391 & 5,753 & 52,144 & 147,516 & 970 & 148,486 \\
\hline 2000-2001 & & & & 130,754 & 849 & 131,603 \\
\hline $2002-2003$ & & & 38,865 & 118,444 & 647 & 119,091 \\
\hline $2004-2005$ & & & & 117,706 & 2,272 & 119,978 \\
\hline$>2006$ & & & 5,488 & & & 141,437 \\
\hline Total & 401,909 & 85,504 & 531,766 & $1,902,734$ & 86,474 & $2,130,645$ \\
\hline
\end{tabular}

and limited pedigree information, especially for non-US genotyped bulls. The difference between UPG for US estimates and non-US sires was smaller 15 yr ago.

Figure 2 shows the same UPG effects as in Figure 1 for dams. The figure shows that the last 7 groups between 1994 and 2006 to focus on the differences. The last original UPG effect for the EBV07 model was underestimated, whereas the last original UPG effect with the GEBV07 model was significantly overestimated by 4.82 and 2.7 times the genetic standard deviation of the final score. The difference between UPG estimates for US and non-US dams was smaller in the early 1990s. Large biases may have occurred in the original UPG for dams because contributions in UPG from their progenies with phenotypes were smaller than those for sires.

The UPG included in MME for any species should be defined based on genetic differences among groups. To estimate the UPG accurately, sufficient numbers of animals and phenotypes for each group are essential. For example, if a small number of animals or no phenotypes are available for one UPG, the UPG estimate could be significantly biased. When some of the pedigrees in foreign or crossbred animals are missing, the bias in UPG may be amplified but could be minimized by simply introducing pedigrees from other countries or crossbred producers.

Figure 3 shows the scatter diagram between GEBV with the original UPG (GEBV07orig) and with the redefined UPG (GEBV07redef1) by combining the last 2 UPG for 2,425 genotyped bulls that had no daughters in 2007 but had at least 10 daughters in 2011. The smaller group of data points indicates inflation of GEBV07orig. Most of these genotyped bulls were found to be sons of 3 non-US sires. Different selection strategies or gene-environment (nation) interaction can

Table 3. Number of US and non-US bulls (\%) with unknown parents

\begin{tabular}{lcc}
\hline $\begin{array}{l}\text { No. of } \\
\text { bulls }\end{array}$ & $\begin{array}{c}\text { US bulls } \\
(\%)\end{array}$ & $\begin{array}{c}\text { Non-US bulls } \\
(\%)\end{array}$ \\
\hline $\begin{array}{l}\text { Missing } \\
\text { Sires }\end{array}$ & $160(0.1)$ & $1,301(14.6)$ \\
Dams & $655(0.3)$ & $1,719(19.3)$ \\
PGS & $341(0.2)$ & $1,455(16.3)$ \\
PGD & $454(0.2)$ & $1,492(16.7)$ \\
MGS & $1,364(0.7)$ & $4,047(45.4)$ \\
MGD & $1,677(0.9)$ & $4,074(45.7)$ \\
Total & 193,716 & 8,915 \\
\hline
\end{tabular}

${ }^{1} \mathrm{PGS}=$ paternal grandsires; $\mathrm{PGD}=$ paternal granddams; MGS $=$ maternal grandsires; MGD = maternal granddams. 


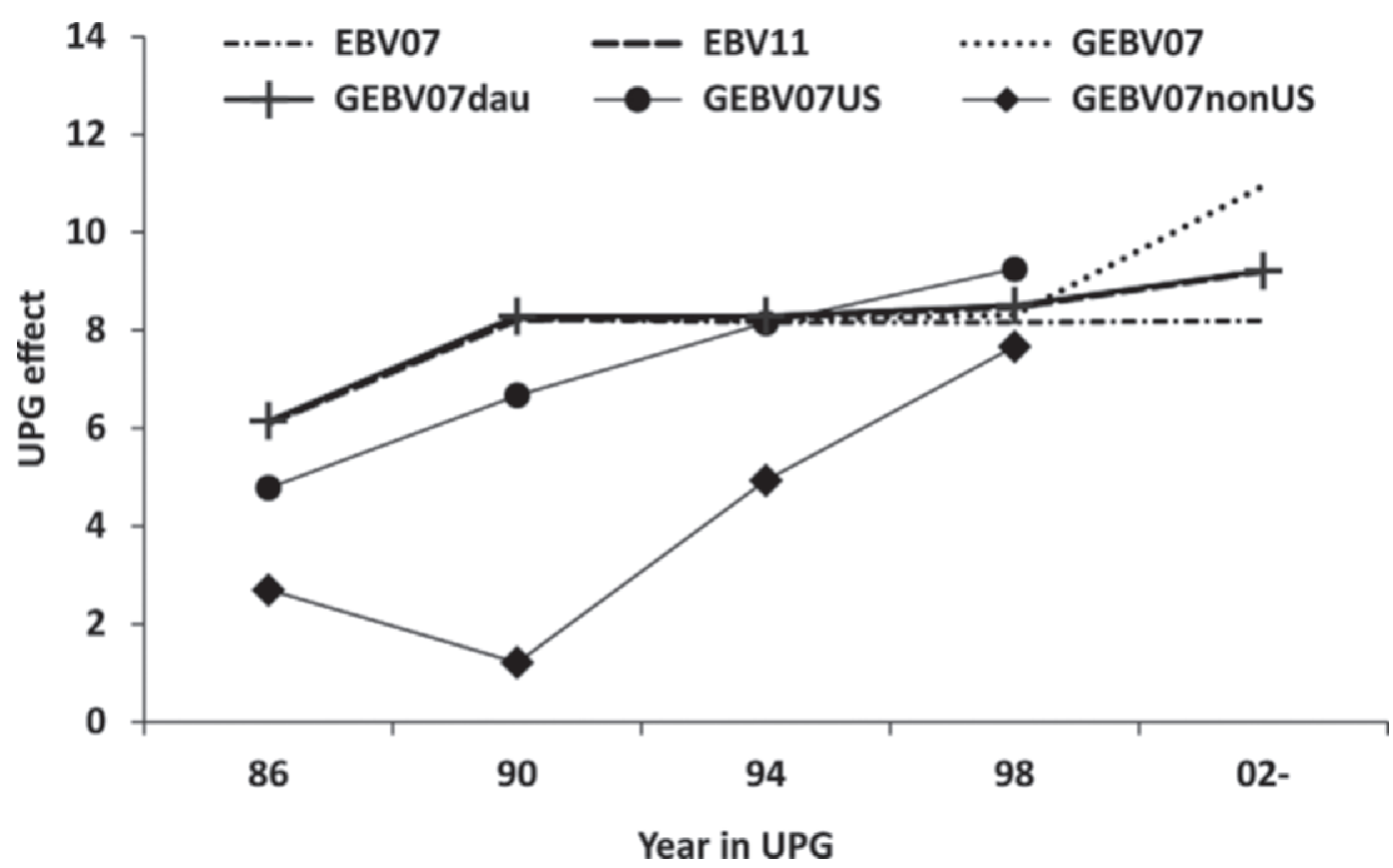

Figure 1. Unknown parent group (UPG) effects for sires in EBV for 2007 data (EBV07), EBV for 2011 data (EBV11), genomic EBV (GEBV) for 2007 data (GEBV07), and GEBV including bulls with daughters (GEBV07dau), GEBV with US bulls (GEBV07US), and GEBV with non-US bulls (GEBV07nonUS).

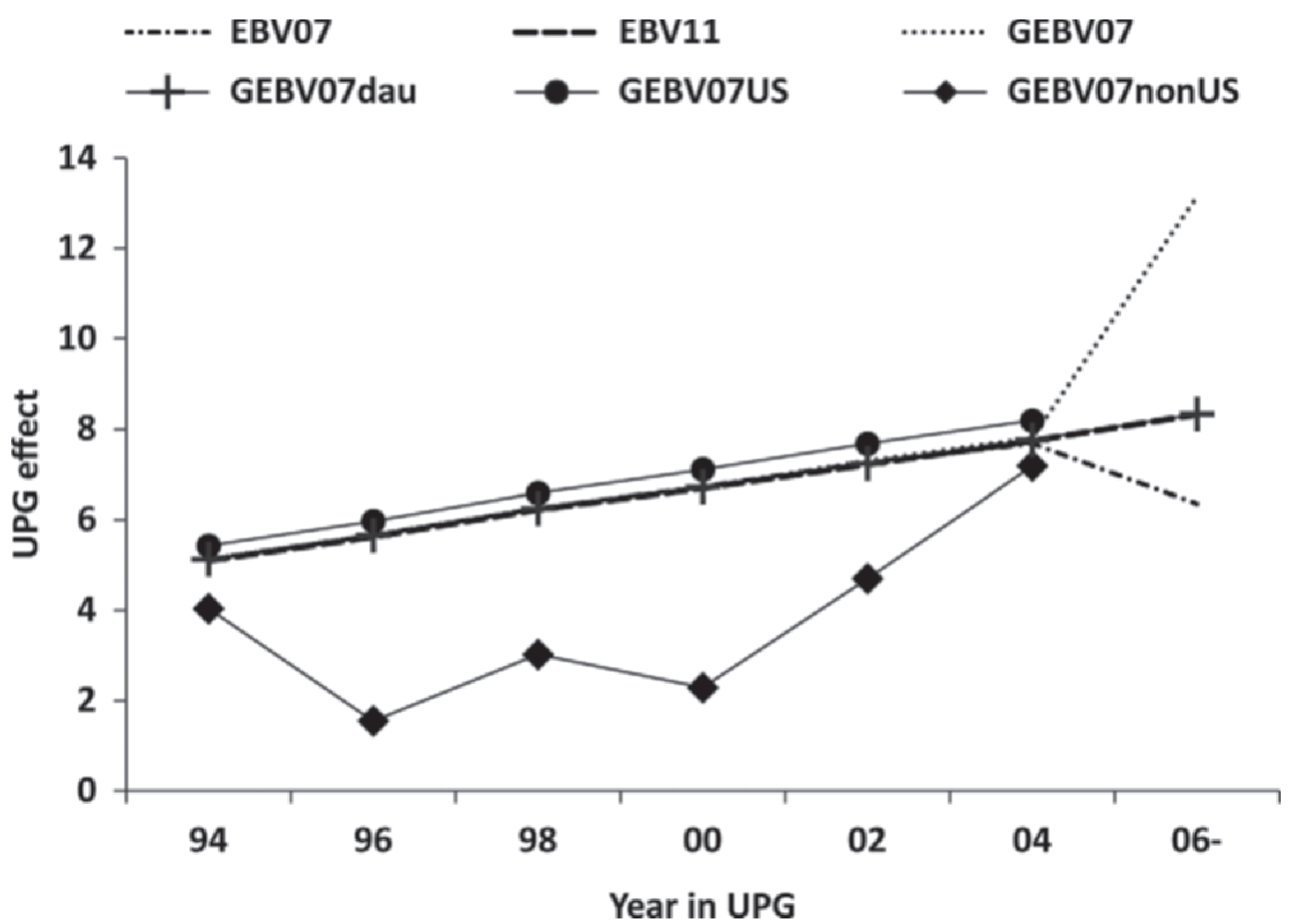

Figure 2. Unknown parent group (UPG) effects for dams in EBV for 2007 data (EBV07), EBV for 2011 data (EBV11), genomic EBV (GEBV) for 2007 data (GEBV07), and GEBV including bulls with daughters (GEBV07dau), GEBV with US bulls (GEBV07US), and GEBV with non-US bulls (GEBV07nonUS). 


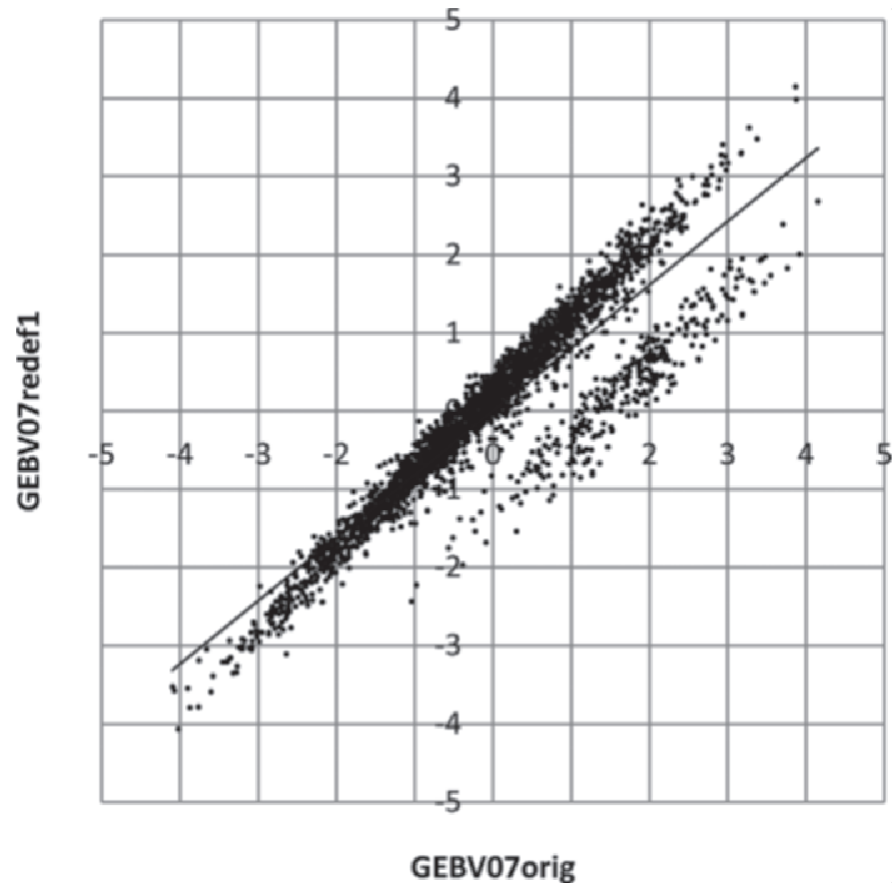

Figure 3. Scatter diagram of genomic EBV (GEBV) with original unknown parent group (UPG) (GEBV07orig) and GEBV with redefined UPG for 2007 data (GEBV07redef1) by combining the last 2 UPG.

create genetic difference among purebred animals from various nations. However, in this study, insufficient dam information of non-US bulls probably created this inflation.

Genetic trends in Figure 4 were plotted by calculating yearly average $(\mathrm{G}) \mathrm{EBV}$ for bulls with at least 1 daughter in 2011, setting the common genetic base to the average (G)EBV of cows born in 2005. Taking UPG into account in MME can also provide unbiased genetic trends by adjusting the genetic base in each UPG. Bulls with at least 1 daughter were used in these calculations to minimize the fluctuation of each line. Genetic trends before 1986 were omitted to focus on those in recent years. Compared with the realized genetic trend in 2011 (EBV11orig), which was assumed to be unbiased, the genetic trend from GEBV07orig was inflated in the last few years, whereas the genetic trend from GEBV07redef1 was deflated. The low genetic trend for GEBV06redef1 in the last few years can be explained by the combined last UPG effect, which was identical to the previous group effect and lower than the last group effect in the GEBV07orig model, as shown in Figure 1. On the other hand, the genetic trend from GEBV07redef2, which combined the last 2 UPG separately for US and non-US unknown parents, was similar to that for EBV11orig, implying less bias in GEBV07redef2. The slope of the genetic trend from
GEBV07noUPG was less steep because the GEBV ignored $\mathbf{Q g}$ in $\mathbf{u}=\mathbf{Q g}+\mathbf{a}$, indicating small or no genetic gains in recent years. Therefore, when genetic improvement is in progress, genetic trends without UPG can be misleading.

Table 4 presents regression coefficients and coefficients of determination of PA in 2007 (PA07) and GEBV07 on progeny deviations in 2011 for 2,425 genotyped bulls with no daughters in 2007 and with at least 10 daughters in 2011. If genetic bases for progeny deviations in 2011 and PA07 or GEBV07 were set similar (e.g., setting the genetic base to the average EBV for cows born in 2005, as shown in Figure 4), the actual bias of PA07 and GEBV07 might be small. The coefficients of determination in PA07 for non-US bulls were significantly lower than those for US bulls. Regression coefficients increased from 0.81 for GEBV07orign to 0.86 for GEBV07redef1 (0.86 and 0.87 for US and non-US bulls, respectively) and to 0.85 for GEBV07redef2 (0.85 and 1.00 for US and non-US bulls, respectively), although Figure 4 shows similar genetic trends for the GEBV07redef2 and EBV11. This small inconsistency between Table 4 and Figure 4 may have occurred because the genetic trends were calculated including genotyped and nongenotyped bulls. Regression coefficients for GEBV07redef1 and GEBV07redef2 were greater than GEBV07noUPG and GEBV07orig, implying that redefining of UPG reduced the bias. The coefficient of determination was lower for GEBV07redef1 but slightly higher for GEBV07redef2 than GEBV07orig. Low regression coefficients $<1.0$ can be improved by using a weight $<1.0$ (e.g., 0.7) on $\mathbf{A}_{22}$ as described in Tsuruta et al. (2011). The low regression coefficients were estimated not only for GEBV but also for PA, suggesting potential biases in EBV without genotypes. Figure 1 might be able to explain the regression coefficient of 1.0 for non-US bulls in GEBV07redef2 because smaller UPG effects for non-US bulls can reduce the inflation in GEBV07redef2 for non-US bulls. However, regression coefficients and coefficients of determination for non-US bulls may not be reliable because of a small number of genotyped bulls (118) compared with the 2,307 US bulls. In addition to the bias described by VanRaden and Wright (2013) attributable to preselection, biases in GEBV for young genotyped bulls could become significant when those bulls have no daughters and the UPG is poorly defined in the model. Rank correlations between EBV11 and GEBV07 were also calculated (Table 4). The rank correlations of EBV11 were lowest with GEBV07noUPG (0.73) and highest with GEBVredef2 (0.86). The rank correlations indicate that $47,36,36$, and $26 \%$ of the young genotyped bulls were inaccurately ranked with GEBV07noUPG, 


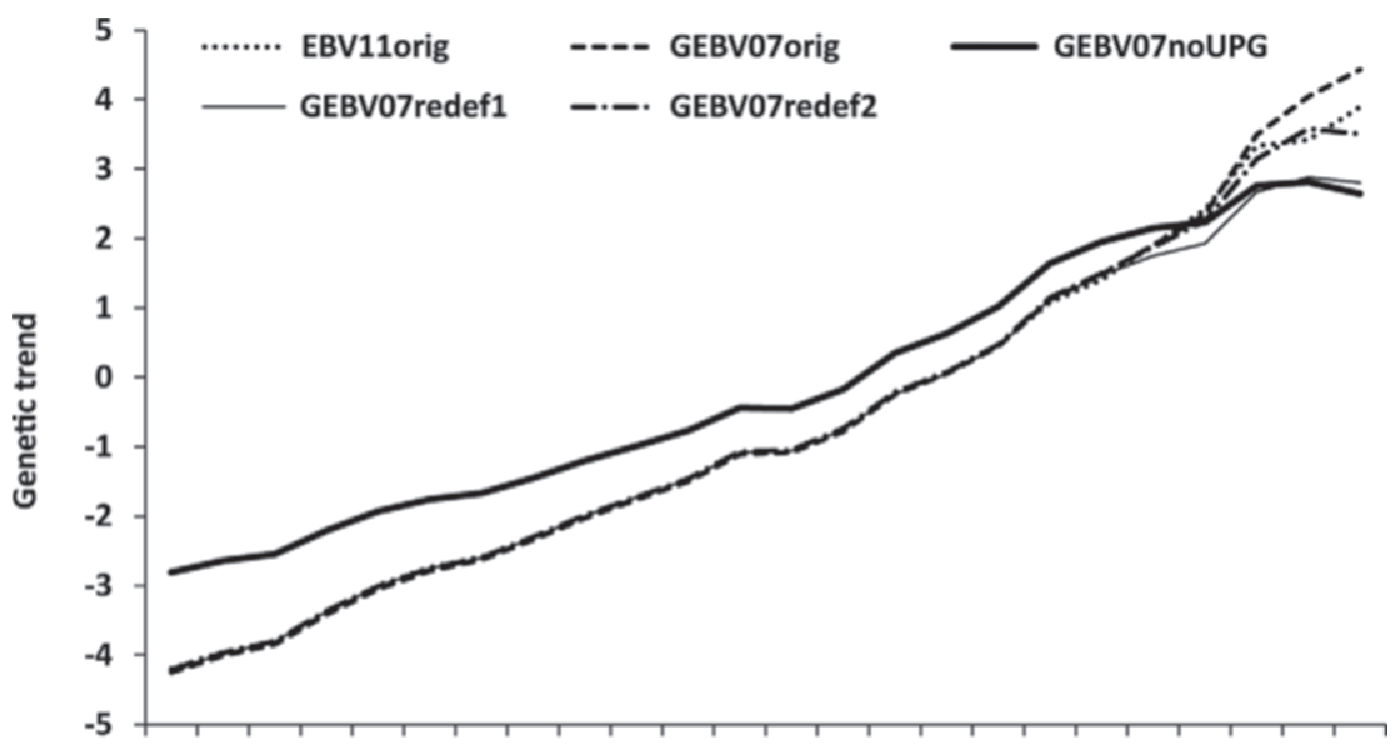

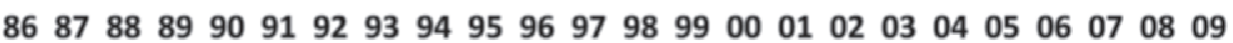

Year of birth

Figure 4. Genetic trends for bulls with at least 1 daughter in 2011 using EBV with original unknown parent group (UPG) for 2011 data (EBV11orig), genomic EBV (GEBV) with original UPG for 2007 data (GEBV07orig), GEBV with no UPG for 2007 data (GEBV07noUPG), and GEBV with redefined UPG for 2007 data by combining the last 2 groups (GEBV07redef1) and by separating for US and non-US bulls (GEBV07redef2) as well as combining.

GEBV07orig, GEBV07redef1, and GEBV07redef2, respectively, when their daughters had no phenotypes.

Generations, sex, regions, selection lines, and origins can cause genetic differentials in a breeding population, and they should be properly defined in genetic groups, especially in genomic evaluations for young genotyped animals. In genomic evaluation, young genotyped animals (especially, young bulls in dairy cattle) are of primary interest. However, young animals that have no phenotypes could cause biases in UPG and GEBV. One reason may be due to no contribution from phenotypes to estimate UPG effects, as described earlier. Another reason may be due to unknown parents of external or foreign animals, especially when they have no phenotypes. One way to account for the missing pedigree information is to incorporate more external sources of

Table 4. Regression coefficients and $\mathrm{R}^{2}(\%)$ of parent average (PA07) and genomic EBV (GEBV07) in 2007 on progeny deviations in 2011 and rank correlations between GEBV07 and genomic EBV in 2011, using different unknown parent group (UPG) definitions (no UPG, original, redefined1 by combining the last 2 groups, and redifined 2 by splitting into US and non-US groups and combining the last 2 groups) for all $(2,425)$, US $(2,307)$, and non-US (118) young genotyped bulls with no daughters in 2007 and with at least 10 daughters in 2011

\begin{tabular}{|c|c|c|c|c|c|c|}
\hline \multirow{2}{*}{$\begin{array}{l}\text { UPG } \\
\text { definition }\end{array}$} & \multirow{2}{*}{$\begin{array}{l}\text { Young } \\
\text { genotyped bulls }\end{array}$} & \multicolumn{2}{|c|}{ Regression } & \multicolumn{2}{|c|}{$\mathrm{R}^{2}$} & \multirow{2}{*}{$\begin{array}{c}\text { Rank } \\
\text { correlation }\end{array}$} \\
\hline & & PA07 & GEBV07 & PA07 & GEBV07 & \\
\hline \multirow[t]{3}{*}{ No UPG } & All & 0.75 & 0.78 & 22 & 24 & 0.73 \\
\hline & US & 0.77 & 0.80 & 23 & 25 & 0.74 \\
\hline & Non-US & 0.57 & 0.66 & 17 & 20 & 0.66 \\
\hline \multirow[t]{3}{*}{ Original } & All & 0.76 & 0.81 & 22 & 33 & 0.80 \\
\hline & US & 0.78 & 0.81 & 23 & 33 & 0.81 \\
\hline & Non-US & 0.65 & 0.86 & 18 & 34 & 0.75 \\
\hline \multirow[t]{3}{*}{ Redefined1 } & All & 0.76 & 0.86 & 22 & 29 & 0.80 \\
\hline & US & 0.78 & 0.86 & 23 & 30 & 0.80 \\
\hline & Non-US & 0.65 & 0.87 & 18 & 28 & 0.84 \\
\hline \multirow[t]{3}{*}{ Redefined2 } & All & 0.76 & 0.85 & 22 & 34 & 0.86 \\
\hline & US & 0.78 & 0.85 & 23 & 34 & 0.86 \\
\hline & Non-US & 0.64 & 1.00 & 17 & 35 & 0.83 \\
\hline
\end{tabular}


information. In cases where this is difficult to get the information, redefining UPG in MME could be a practical method in genomic evaluations and may be useful for any breed and species.

\section{CONCLUSIONS}

Poorly defined unknown parent groups could provide unreliable breeding values and misleading genetic trends. Incorrect UPG estimates in single-step genomic evaluations could increase bias and decrease accuracy in genomic EBV. Minimizing the bias in genomic evaluations of young genotyped animals is crucial because they are the most valuable resources for genomic selection. When defining UPG in genomic evaluation, each unknown parent group must contain a sufficient number of animals and some of those animals should have phenotypes to share the contribution with the UPG for young genotyped animals with no phenotypes. Moreover, when external or foreign pedigree information of young genotyped animals is not available, assigning separated UPG for internal (domestic) and external (foreign) animals may be useful.

\section{ACKNOWLEDGMENTS}

This study was partially funded by Holstein Association USA Inc. (Brattleboro, VT). The authors thank Holstein Association USA Inc. and USDA Agricultural Research Service-Animal Improvement Programs Laboratory (ARS-AIPL) for providing Holstein final score data with pedigree and genomic data.

\section{REFERENCES}

Aguilar, I., I. Misztal, D. L. Johnson, A. Legarra, S. Tsuruta, and T. J. Lawlor. 2010. Hot topic: A unified approach to utilize phenotypic, full pedigree, and genomic information for genetic evaluation of Holstein final score. J. Dairy Sci. 93:743-752.

Henderson, C. R. 1974. Sire evaluation and genetic trends. Pages 1041 in Proc. Anim. Breed. Genet. Symp. in Honor of Dr. Jay L. Lush. American Society of Animal Science (ASAS) and American Dairy Science Association (ADSA), Champaign, IL.

Interbull. 2001. Interbull Guidelines for National \& International Genetic Evaluation Systems in Dairy Cattle with Focus on Production Traits. Interbull Bull. 28. Interbull, Uppsala, Sweden.

Kennedy, B. W. 1981. Bias and mean square error from ignoring genetic groups in mixed model sire evaluation. J. Dairy Sci. 64:689697.

Mäntysaari, E., Z. Liu, and P. VanRaden. 2010. Interbull validation test for genomic evaluations. Interbull Bull. 41:17-22.

Misztal, I., Z. G. Vitezica, A. Legarra, I. Aguilar, and A. A. Swan. 2013. Unknown-parent groups in single-step genomic evaluation. J. Anim. Breed. Genet. 130:252-258.

Quaas, R. L., and E. J. Pollak. 1981. Modified equations for sire models with groups. J. Dairy Sci. 64:1868-1872.

Tsuruta, S., D. A. L. Lourenco, and I. Misztal. 2013. Bias in singlestep genomic evaluations attributable to unknown parent group estimates. J. Dairy Sci. 96(E-Suppl. 1):75. (Abstr.)

Tsuruta, S., I. Misztal, I. Aguilar, and T. J. Lawlor. 2011. Multipletrait genomic evaluation of linear type traits using genomic and phenotypic data in Holsteins. J. Dairy Sci. 94:4198-4204.

Tsuruta, S., I. Misztal, L. Klei, and T. J. Lawlor. 2002. Analysis of agespecific predicted transmitting abilities for final scores in Holsteins with a random regression model. J. Dairy Sci. 85:1324-1330.

Tsuruta, S., I. Misztal, and I. Strandén. 2001. Use of the preconditioned conjugate gradient algorithm as a generic solver for mixed model equations in animal breeding applications. J. Anim. Sci. 79:1166-1172.

VanRaden, P. M., C. P. Van Tassell, G. R. Wiggans, T. S. Sonstegard, R. D. Schnabel, J. F. Taylor, and F. S. Schenkel. 2009. Invited review: Reliability of genomic predictions for North American Holstein bulls. J. Dairy Sci. 92:16-24.

VanRaden, P. M., and J. R. Wright. 2013. Measuring genomic preselection in theory and in practice. Interbull Bull. 47:147-150.

Westell, R. A., R. L. Quaas, and L. D. Van Vleck. 1988. Genetic groups in an animal model. J. Dairy Sci. 71:1310-1318.

Wiggans, G. R., P. M. VanRaden, and T. A. Cooper. 2011. The genomic evaluation system in the United States: Past, present, future. J. Dairy Sci. 94:3202-3211. 